VOL. 43 (1991) [269-272]

\title{
A PROPERTY OF SUBGROUPS OF FREE GROUPS
}

\section{Gerhard ROSENBERger}

Dedicated to B.H. Neumann for his 80th Birthday

\section{INTRODUCTION}

Let $F$ be a free group on $a_{1}, \ldots, a_{p}(p \geqslant 1)$, and $X$ a finitely generated subgroup in $F$. Suppose either that $X$ contains some non-trivial power of $a_{1}^{2} \ldots a_{p}^{2}$, or that $p$ is even and $X$ contains some nontrivial power of $\left[a_{1}, a_{2}\right] \ldots\left[a_{p-1}, a_{p}\right]$. We discuss some properties of $X$ which we can derive from this assumption.

In this note we use the terminology and notation of $[2,4,6]$; here $\langle\ldots \mid \ldots\rangle$ denotes a presentation of a group in terms of generators and relations. By $\left\langle b_{1}, \ldots, b_{n}\right\rangle$ we denote the group generated by $b_{1}, \ldots, b_{n} ;[a, b]=a b a^{-1} b^{-1}$ is the commutator of $a, b \in G$ ( $G$ a group). Frequently we obtain from one system $\left\{x_{1}, \ldots, x_{m}\right\}$ a new one by Nielsen transformations, and then denote the latter by the same symbols.

\section{AN EQUATION IN A FREE GROUP}

THEOREM 1. Let $F$ be a free group on $a_{1}, \ldots, a_{p}(p \geqslant 1, p$ even) and $P\left(a_{1}, \ldots, a_{p}\right)=\left[a_{1}, a_{2}\right] \ldots\left[a_{p-1}, a_{p}\right] \in F$. Let $\left(x_{1}, \ldots, x_{m}\right)(m \geqslant 1)$ be any finite sequence of elements of $F$ and $X$ be the subgroup of $F$ generated by $x_{1}, \ldots, x_{m}$. Suppose that $X$ contains some conjugate of some non-trivial power of $P\left(a_{1}, \ldots, a_{p}\right)$, and let $\beta$ be the smallest positive integer such that some conjugate of $P^{\beta}\left(a_{1}, \ldots, a_{p}\right)$ lies in $X$. Then

(a) $\left(x_{1}, \ldots, x_{m}\right)$ is Nielsen equivalent to a free basis for $X$ which contains a conjugate of $P^{\beta}\left(a_{1}, \ldots, a_{p}\right)$; or

(b) the index of $X$ in $F$ is $\beta$ (and $\left\{1, P\left(a_{1}, \ldots, a_{p}\right), \ldots, P^{\beta-1}\left(a_{1}, \ldots, a_{p}\right)\right\}$ is a set of coset representatives for $X$ in $F$ ).

Proof: In the following let $\alpha$ be the smallest positive number for which $y^{-1} p^{\alpha}\left(a_{1}, \ldots, a_{p}\right) y \in X$ for some $y \in F$. If $\alpha=1$ then the statement holds by Theorem (2.2) of [5]. Hence, from now on let $\alpha \geqslant 2$. We may assume that $y=1$ (by replacing the $x_{i}$ by $y x_{i} y^{-1}$ if necessary).

Received 20 April 1990

Copyright Clearance Centre, Inc. Serial-fee code: 0004-9729/91 \$A2.00+0.00. 
By means of the classical Nielsen reduction method we obtain from $\left(x_{1}, \ldots, x_{m}\right)$ a system $\left(y_{1}, \ldots, y_{k}\right), 1 \leqslant k \leqslant m$, which generates $X$ freely and has the Nielsen property, so that in particular, as a freely reduced word in $a_{1}, \ldots, a_{p}$, each $y_{i}$ contains an uncancellable symbol $a_{j}^{e}, \varepsilon= \pm 1$, in the sense that in reducing any product $y_{r}^{e_{r}} y_{s}^{\varepsilon_{s}}, \varepsilon_{r}, \varepsilon_{s}= \pm 1$, these symbols cancel if and only if $r=s$ and $\varepsilon_{r}=-\varepsilon_{d}$. For each $y_{i}$ we choose such a symbol and take the inverse symbol for $y_{i}^{-1}$.

We have an equation

$$
\begin{gathered}
\prod_{i=1}^{q} y_{\nu_{i}}^{\varepsilon_{i}}=P^{\alpha}\left(a_{1}, \ldots, a_{p}\right), \quad \varepsilon_{i}= \pm 1 \\
\varepsilon_{i}=\varepsilon_{i+1} \text { if } \nu_{i}=\nu_{i+1} .
\end{gathered}
$$

If a factor $y_{\nu_{i}}^{\varepsilon_{t}}$ occurs twice in (1) then we have a partial product $\prod_{i=t}^{\infty} y_{\nu_{i}}^{\varepsilon_{i}}$ with $\nu_{t}=$ $\nu_{s+1}, \varepsilon_{t}=\varepsilon_{s+1}$ and no $y_{\nu_{i}}^{e_{i}}$ occurring twice in $y_{\nu_{t}}^{e_{t}} \ldots y_{\nu_{t}}^{v e_{s}}$. The symbol of $y_{\nu_{t}}^{e_{t}}$ occurs twice in $\prod_{i=1}^{q} y_{\nu_{i}}^{\varepsilon_{i}}$, and also in $P^{\alpha}\left(a_{1}, \ldots, a_{p}\right)$. Hence $\prod_{i=t}^{v_{i}} y_{\nu_{i}}^{\varepsilon_{i}}$ is conjugate to a power of $P\left(a_{1}, \ldots, a_{p}\right)$. Therefore, after a suitable conjugation, we obtain an equation which may be assumed to have the form

$$
\prod_{i=1}^{r} y_{\nu_{i}}^{e_{i}}=P^{\beta}\left(a_{1}, \ldots, a_{p}\right), 1 \leqslant \beta \leqslant \alpha, \varepsilon_{i}= \pm 1, \varepsilon_{i}=\varepsilon_{i+1} \text { if } \nu_{i}=\nu_{i+1},
$$

with no factor $y_{\nu_{i}}^{\varepsilon_{i}}$ occurring twice (or course we must then in fact have $\alpha=\beta$ because $\alpha$ is minimal). If some $y_{i}$ occurs just once in (2) either with exponent +1 or with exponent -1 then case (a) occurs. Now, let in (2) each $y_{i}$ occur exactly once with exponent +1 and once with exponent -1 . (It can be shown - we omit the details - that every $y_{i}$ must occur in (2).) This implies in particular that $X$ is not cyclic, since $\beta \geqslant 1$. Hence $k \geqslant 2$, and by $[6,5.2]$ we may apply Nielsen transformations for quadratic words to get from $\left(y_{1}, \ldots, y_{k}\right)$ a system $\left(z_{1}, \ldots, z_{k}\right)$ such that

$$
\prod_{i=1}^{r} y_{\nu_{i}}^{e_{i}}=\left[z_{1}, z_{2}\right] \ldots\left[z_{\ell-1}, z_{\ell}\right] z_{\ell+1} z_{\ell+1}^{-1} \ldots z_{k} z_{k}^{-1}, 2 \leqslant \ell \leqslant k, \quad \ell \text { even. }
$$

Without loss of generality, we may assume that $\ell=k$ and, hence, that $k$ is even and $\left(z_{1}, \ldots, z_{k}\right)$ is a free generating system of $X$.

We want to show that $X$ has the finite index $[F: X]=\beta$. From Theorem (2.2) of [5] we know that $F=\left\langle z_{1}, \ldots, z_{k}, P\left(a_{1}, \ldots, a_{p}\right)\right\rangle$. Now we regard $F$ as a cycloidal Fuchsian group of the first kind such that

(i) $a_{1}, \ldots, a_{p}$ are hyperbolic elements and $p / 2$ is the genus of $F$;

(ii) $a=P\left(a_{1}, \ldots, a_{p}\right)^{-1}$ is a parabolic element; and

(iii) $\left\{a, a_{1}, \ldots, a_{p}\right\}$ is a canonical generating system of $F$. 
Now $X$ is, as a subgroup of $F$, also discrete. Moreover, $X$ has finite index in $F$. The essential reasons for this are as follows. Since $F$ is residually finite, for a suitable large integer $\gamma$, the image $X(n)$ of $X$ has finite index $[F(n): X(n)] \leqslant N$ in the factor group $F(n)=\left\langle a_{1}, \ldots, a_{p} \mid\left(P\left(a_{1}, \ldots, a_{p}\right)\right)^{\beta \gamma n}=1\right\rangle,(n \in N)$, of $F$, where $N$ is an integer which does not depend on $n$, and the set of coset representatives for $X(n)$ in $F(n)$ can be chosen independently of $n$ by $[6,4.14]$ and since $F=\left\langle z_{1}, \ldots, z_{k}, P\left(a_{1}, \ldots, a_{p}\right)\right\rangle$. Recalling that a finitely generated subgroup of infinite index in $F(n)$ is automatically a free product of cyclic groups ([1]), it follows by letting $n$ increase that $X$ has finite index $[F: X] \leqslant N$ in $F$. (See also [3, Theorem 2.12].)

In a finitely generated free group no power of a primitive element is contained in its commutator subgroup. Hence, since the index $[F: X]$ is finite, no primitive element of $X$ is conjugate in $F$ to a power of $P\left(a_{1}, \ldots, a_{p}\right)$, and $X$ is a cycloidal Fuchsian group of the first kind such that

(i) $z_{1}, \ldots, z_{k}$ are hyperbolic elements and $k / 2$ is the genus of $X$,

(ii) $b=a^{\beta}=P\left(a_{1}, \ldots, a_{p}\right)^{-\beta}$ is a parabolic element, and

(iii) $\left\{b, z_{1}, \ldots, z_{k}\right\}$ is a canonical generating system of $X$.

If $[F: X]=1$ then $\beta=1$ and $F=X$. Now let $[F: X]>1$, $g \in F \backslash X$, and consider $g P\left(a_{1}, \ldots, a_{p}\right) g^{-1}$. There exists a natural number $\gamma$ such that $g P^{\gamma}\left(a_{1}, \ldots, a_{p}\right) g^{-1} \in X$ since $[F: X]$ is finite. Hence, there exists $h \in X$ and an integer $\delta \neq 0$ such that $h g p^{\gamma}\left(a_{1}, \ldots, a_{p}\right) g^{-1} h^{-1}=p^{\delta}\left(a_{1}, \ldots, a_{p}\right)$, because $X$ is cycloidal. Therefore $\gamma=\delta$ and $h g \in\left\langle P\left(a_{1}, \ldots, a_{p}\right)\right\rangle$ since $F$ is free. Now $g=d P^{\varphi}\left(a_{1}, \ldots, a_{p}\right)$, (whence $X g=X P^{\varphi}\left(a_{1}, \ldots, a_{p}\right)$ ) for some $d \in X$ and some natural number $\varphi$ with $1 \leqslant \varphi<\beta$, since $h \in X$ and $P^{\beta}\left(a_{1}, \ldots, a_{p}\right) \in X$.

Corollary 1. Let $F$ be a free group on $a_{1}, \ldots, a_{p}(p \geqslant 1, p$ even) and $P\left(a_{1}, \ldots, a_{p}\right)=\left[a_{1}, a_{2}\right] \ldots\left[a_{p-1}, a_{p}\right] \in F$. Let $\alpha \in \mathrm{N}, \alpha \geqslant 2$, and $x_{1}, y_{1}, \ldots, x_{q}, y_{q} \in F(q \geqslant 1)$ be such that $P^{\alpha}\left(a_{1}, \ldots, a_{p}\right)=\prod_{i=1}^{q}\left[x_{i}, y_{i}\right]$. Suppose that $P^{\alpha}\left(a_{1}, \ldots, a_{p}\right)$ is not a proper power in the subgroup $X$ generated by $x_{1}, y_{1}, \ldots, x_{q}, y_{q}$. Then $\alpha$ is odd and $q \geqslant(\alpha(p-1)+1) / 2$.

Proof: Note that $X$ has finite index $\alpha$ in $F$ by Theorem 1. If we regard $F$ as a cycloidal Fuchsian group of the first kind such that $P\left(a_{1}, \ldots, a_{p}\right)$ is parabolic then the Riemann-Hurwitz relation yields that $\alpha$ is odd and $\alpha(p-1) \leqslant 2 q-1$, giving the result.

If we regard a free group $F$ on $a_{1}, \ldots, a_{p}(p \geqslant 1)$ as a plane discontinuous group $F=\left\langle a, a_{1}, \ldots, a_{p} \mid a a_{1}^{2} \ldots a_{p}^{2}=1\right\rangle$ we get analogously the following,

ThEOREM 2. Let $F$ be a free group on $a_{1}, \ldots, a_{p}(p \geqslant 1)$ and let $Q\left(a_{1}, \ldots, a_{p}\right)=$ 
$a_{1}^{2} \ldots a_{p}^{2} \in F$. Let $\left(x_{1}, \ldots, x_{m}\right)(m \geqslant 1)$ be any finite sequence of elements of $F$ and $X$ be the subgroup of $F$ generated by $x_{1}, \ldots, x_{m}$. Suppose that $X$ contains some conjugate of some nontrivial power of $Q\left(a_{1}, \ldots, a_{p}\right)$, and let $\beta$ be the smallest positive integer such that some conjugate of $Q^{\beta}\left(a_{1}, \ldots, a_{p}\right)$ lies in $X$. Then:

(a) $\left\{x_{1}, \ldots, x_{m}\right\}$ is Nielsen equivalent to a free basis for $X$ which contains a conjugate of $Q^{\beta}\left(a_{1}, \ldots, a_{p}\right)$; or

(b) the index of $X$ in $F$ is $\beta$, (and $\left\{1, Q\left(a_{1}, \ldots, a_{p}\right), \ldots, Q^{\beta-1}\left(a_{1}, \ldots, a_{p}\right)\right\}$ is a set of coset representatives for $X$ in $F$ ).

CoROLlary 2. Let $F$ be a free group on $a_{1}, \ldots, a_{p}(p \geqslant 1)$ and $Q\left(a_{1}, \ldots, a_{p}\right)=$ $a_{1}^{2} \ldots a_{p}^{2} \in F$. Let $\alpha \in N, \alpha \geqslant 2$, and $x_{1}, \ldots, x_{q} \in F(q \geqslant 1)$ be such that

$$
Q^{\alpha}\left(a_{1}, \ldots, a_{p}\right)=x_{1}^{2} \ldots x_{q}^{2} .
$$

Then if $Q^{\alpha}\left(a_{1}, \ldots, a_{p}\right)$ is not a proper power in the subgroup $X$ generated by $x_{1}, \ldots, x_{q}$, we have $q \geqslant \alpha(p-1)+1$.

\section{REFERENCES}

[1] A.H.M. Hoare, A. Karrass and D. Solitar, 'Subgroups of infinite index in Fuchsian groups', Math. Z. 125 (1972), 59-69.

[2] R.C. Lyndon and P.E. Schupp, Combinatorial Group Theory 189, (Ergebnisse der Mathematik) (Springer-Verlag, Berlin, Heidelberg, New York, 1977).

[3] W. Magnus, A. Karrass and D. Solitar, Combinatorial Group Theory: Presentations of Groups in Terms of Generators and Relations: Interscience Tracts in Pure and Applied Mathematics 20 (Wiley, New York, 1966).

[4] G. Rosenberger, 'Über Darstellungen von Elementen und Untergruppen in freien Produkten', in Proceedings of 'Groups-Korea 1983': Lecture Notes in Mathematics 1098, pp. 142-160 (Springer-Verlag, Berlin, Heidelberg, New York, 1984).

[5] G. Rosenberger, 'Minimal generating systems for plane discontinuous groups and an equation in free groups', in Proceedings of 'Groups-Korea 1988': Lecture Notes in Mathematics 1398, pp. 170-186 (Springer-Verlag, Berlin, Heidelberg, New York, 1989).

[6] H. Zieschang, E. Vogt and H.-D. Coldewey, 'Surfaces and Planar Discontinuous Groups', in Lecture Notes in Mathematics 835 (Springer-Verlag, Berlin, Heidelberg, New York, 1980).

Fachbereich Mathematik

Universität Dortmund

Postfach 500500

Federal Republic of Germany 